\title{
Evidence for an oscillation of the magnetic axis of the white dwarf in the polar DP Leonis
}

\author{
K. Beuermann ${ }^{1}$, S. Dreizler ${ }^{1}$, F. V. Hessman ${ }^{1}$, and A. D. Schwope ${ }^{2}$ \\ ${ }^{1}$ Institut für Astrophysik, Georg-August-Universität, Friedrich-Hund-Platz 1, 37077 Göttingen, Germany \\ e-mail: beuermann@astro.physik.uni-goettingen.de \\ ${ }^{2}$ Leibniz-Institut für Astrophysik Potsdam (AIP), An der Sternwarte 16, 14482 Potsdam, Germany
}

Received 4 December 2013 / Accepted 31 December 2013

\begin{abstract}
From 1979 to 2001, the magnetic axis of the white dwarf in the polar DP Leo slowly rotated by $50^{\circ}$ in azimuth, possibly indicating a small asynchronism between the rotational and orbital periods of the magnetic white dwarf. Using the MONET/North telescope, we have obtained phase-resolved orbital light curves between 2009 and 2013, which show that this trend has not continued in recent years. Our data are consistent with the theoretically predicted oscillation of the magnetic axis of the white dwarf about an equilibrium orientation, which is defined by the competition between the accretion torque and the magnetostatic interaction of the primary and secondary star. Our data indicate an oscillation period of $\sim 60 \mathrm{yr}$, an amplitude of about $25^{\circ}$, and an equilibrium orientation leading the connecting line of the two stars by about $7^{\circ}$.
\end{abstract}

Key words. binaries: close - binaries: eclipsing - white dwarfs - novae, cataclysmic variables - stars: individual: DP Leonis

\section{Introduction}

Since its discovery by Biermann et al. (1985), the 18 mag shortperiod magnetic cataclysmic variable or polar DPLeo $\left(P_{\text {orb }}=\right.$ $89.8 \mathrm{~min}$ ) has shown an accretion geometry, characterized by a prime accreting pole that points approximately toward the secondary and a weakly accreting pole in the opposite hemisphere. The former is responsible for the optical cyclotron and X-ray emission in the "bright phase", which lasts slightly longer than half the orbital period (Schaaf et al. 1987; Bailey et al. 1993; Robinson \& Cordova 1994; Pandel et al. 2002; Schwope et al. 2002). The latter usually emits only faint X-ray and cyclotron radiation with prominent cyclotron emission lines. The magnetic field strengths are $30.5 \mathrm{MG}$ and $59 \mathrm{MG}$ in the accretion regions near the prime and second pole, respectively (Cropper \& Wickramasinghe 1993).

The mechanism responsible for the attainment of synchronism in polars, that is, the equality of rotational and orbital periods of the white dwarf, is thought to be the interaction between the magnetic moments of primary and secondary. Synchronism is attained if the magnetic torque dominates the accretion torque. A variable accretion torque or some other perturbation may lead to an oscillation (or libration) of the magnetic axis about its equilibrium position, measured as a secular variation of the longitude of the accretion spot. The predicted period of such an oscillation is of the order of $50 \mathrm{yr}$ (King et al. 1990; King \& Whitehurst 1991; Wickramasinghe \& Wu 1991; Wu \& Wickramasinghe 1993; Campbell \& Schwope 1999). If equilibrium is not attained, the white dwarf may rotate slowly under the action of the accretion torque. Four polars lack strict synchronization, displaying a difference between rotational and orbital periods on the order of 1\% (Campbell \& Schwope 1999).
The timing observations of DP Leo available until 2001 were summarized by Pandel et al. (2002) and Schwope et al. (2002). They noted that between 1979 and 2001, the longitude of the accretion spot increased linearly by about $2.3^{\circ}$ per year, implying a rotation period shorter than the orbital period by $5.9 \mathrm{~ms}$. At the same time, the $\mathrm{O}-\mathrm{C}$ variations of the binary were interpreted as a monotonic decrease of the orbital period by $5.3 \times 10^{-12} \mathrm{~s} \mathrm{~s}^{-1}$, suggesting that the binary was heading toward synchronism on a time scale of $\sim 30 \mathrm{yr}$. This interpretation appeared plausible since the polar V1500 Cyg displayed a similar behavior after having suffered an outburst as Nova Cyg 1975, in which it lost synchronism (Schmidt \& Stockman 1991). A decade later, however, Qian et al. (2010) and Beuermann et al. (2011) demonstrated that a monotonous period decrease in DP Leo does not exist and reinterpreted the $\mathrm{O}-\mathrm{C}$ variations as a light travel time effect caused by a giant planet orbiting the binary, while the binary period remained constant. This again raises the question of the origin of the secular variation of the spot longitude.

In this paper, we report new measurements which show that the progression of the spot longitude has halted since 2009. Our data are consistent with an oscillation of the magnetic axis of the white dwarf around its equilibrium position, placing the interpretation of the DP Leo data on a new basis.

\section{Observations}

We observed the cataclysmic variable DPLeo in 45 nights between March 2009 and March 2013, using the $1.2 \mathrm{~m}$ MONET/North telescope at the University of Texas McDonald Observatory via the MONET browser-based remote-observing interface. Photometric light curves in white light were obtained, using an Apogee ALTA E47+ 1k $\times 1 \mathrm{k}$ CCD camera. Exposure 


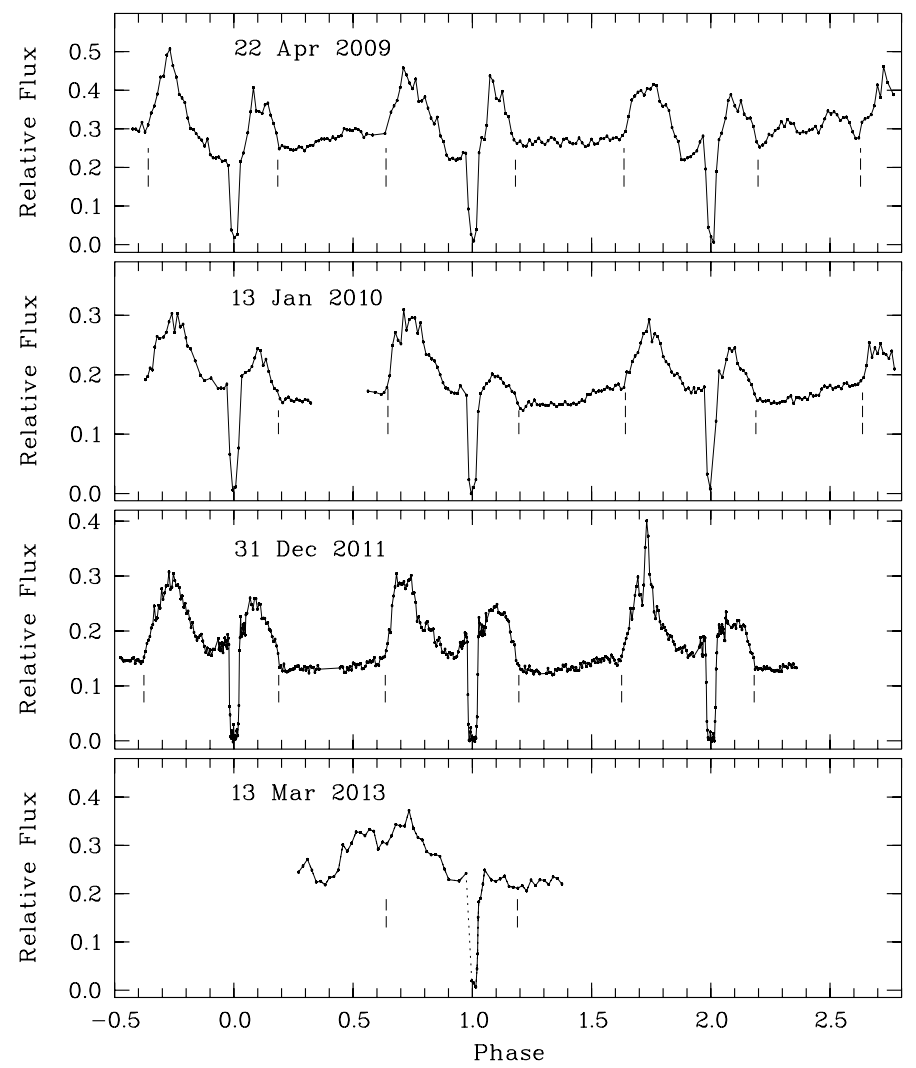

Fig. 1. Examples of the light curves of DP Leo taken in white light between March 2009 and March 2013, phased relative to the eclipse of the white dwarf by the secondary star. The vertical dashed markers indicate the beginning and end of the visibility of the prime accretion spot (see text).

times of $30 \mathrm{~s}$ or $60 \mathrm{~s}$ were use for the orbital light curves, the narrow eclipses were covered with $10 \mathrm{~s}$ exposures, all separated by $3 \mathrm{~s}$ readout. Photometry was performed relative to the $r=16.86$ comparison star SDSS J111725.54+175614.9, which is located $2.29 \mathrm{E}$ and $1.45 \mathrm{~S}$ of the binary and has colors that approximate those of DPLeo. The images were corrected for dark current and flatfielded in the usual way. Fluxes were extracted, using a radius of about one FWHM of the point-spread function.

The mid-eclipse times of the white dwarf, which define orbital phase $\phi=0$, can be determined from the observed light curves with an accuracy better than a few seconds (Beuermann et al. 2011). The shutter times and the time stamps of the MONET/North telescope have an accuracy of a small fraction of a second, more than adequate for the present purpose. We converted the measured times from UTC to barycentric dynamical time (TDB) and corrected them for the light travel time to the solar system barycenter, using the tool provided by Eastman et al. $(2010)^{1}$. The linear ephemeris of Beuermann et al. (2011, their Eq. (1)) was found to be still valid within a few seconds in early 2013. We conclude that the orbital-phase error for a given measured UTC timing is less than 0.001. The new eclipse data will be reported elsewhere.

\footnotetext{
1 http://astroutils.astronomy.ohio-state.edu/time/
}
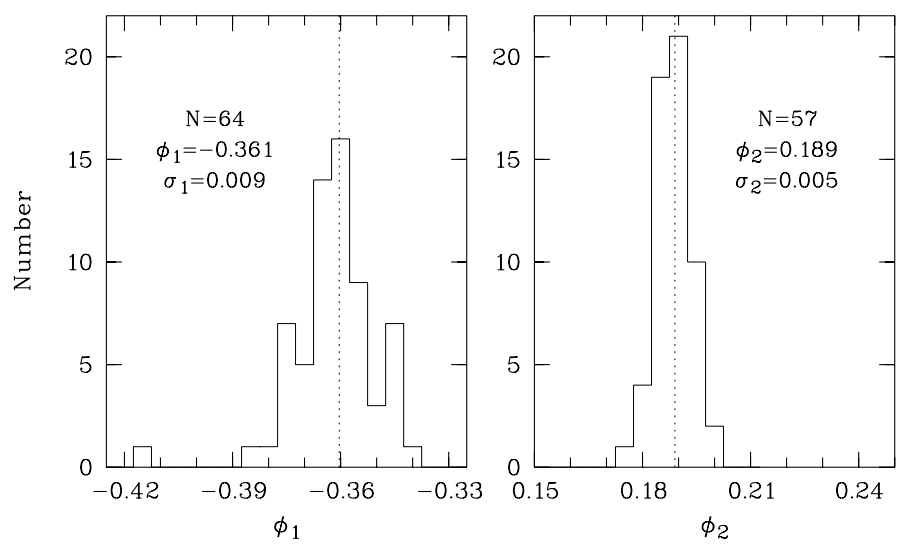

Fig. 2. Distributions of the measured phases of the beginning (left panel) and end (right panel) of the visibility of the prime accretion spot.

\section{Results}

\subsection{Orbital light curves}

Figure 1 shows examples of the total of 45 white-light orbital light curves of DP Leo taken in five observing seasons between March 2009 to March 2013². All light curves are phased relative to the simultaneously observed eclipses of the white dwarf by the secondary star (Beuermann et al. 2011). The general shape of the light curve is characterized by a bright phase, which extends from about $\phi \simeq-0.36$ to 0.19 and is caused by emission from the prime pole visible during this interval (Pandel et al. 2002; Schwope et al. 2002, and references therein). Cyclotron beaming is responsible for the double-humped shape.

The flux in the phase interval, $\phi=0.19-0.64$, is cyclotron emission from the usually fainter second pole with a small contribution from the white dwarf photosphere (except for a low state, when the cyclotron emission disappears). A doublehumped structure is also observed in a few instances in the faint phase. This is a transient phenomenon, as seen in the top panel of Fig. 1, where maxima at orbital phases of about 0.30 and 0.50 appear in the third of three faint-phase intervals, but these maxima are absent from the second interval. Traces appear frequently, in particular of the $\phi=0.50$ hump. On a single occasion, on 13 March 2013, the $\phi=0.50$ hump rivaled the prime-pole emission in brightness (Fig. 1, bottom panel), rendering the beginning of the prime-pole bright phase unmeasurable.

The vertical dashed markers in the top three panels of Fig. 1 indicate the individual measurements of the beginning and end of the visibility of the prime-pole accretion spot, $\phi_{1}$ and $\phi_{2}$, respectively, in the bottom panel they refer to the expected phases. All light curves combined yield a total of 64 measurements of $\phi_{1}$ and 57 of $\phi_{2}$. Figure 2 shows the histograms of all $\phi_{1}$ and $\phi_{2}$ measurements collected into phase bins of width 0.005 . The mean values for all five observing seasons combined are $\left\langle\phi_{1}\right\rangle=0.361$ and $\left\langle\phi_{2}\right\rangle=0.189$, with standard deviations of the distributions $\sigma_{1}=0.009$ and $\sigma_{2}=0.005$, respectively. With exposure times of $30 \mathrm{~s}$ or $60 \mathrm{~s}$ in the individual light curves (not considered separately) and an orbital period of $5388 \mathrm{~s}$, the minimal timing error, taken as 0.5 bin widths, is between 0.003 and 0.006 phase units. Noise in the light curves may increase this uncertainty. The standard deviation of $\phi_{2}$ is consistent with being entirely due to measurement errors. The $\phi_{1}$ distribution is wider, indicating some in-

\footnotetext{
2 Spring 2009, winter/spring 2009/2010, winter/spring 2010/2011, winter/spring 2011/2012, and early 2013 .
} 
K. Beuermann et al.: Oscillation of the magnetic axis of the white dwarf in DP Leo

Table 1. Longitude $\psi$ of the center of the prime-pole bright phase, based on $n_{1}$ and $n_{2}$ measurements of the beginning and end phases of the bright-pole visibility interval, $\phi_{1}$ and $\phi_{2}$, respectively, in five observing seasons.

\begin{tabular}{lcrcccc}
\hline \hline $\begin{array}{l}\text { Observing } \\
\text { season }\end{array}$ & $\begin{array}{c}\text { Mean JD } \\
\text { 2450 000+ }\end{array}$ & $n_{1}$ & $\phi_{1}$ & $n_{2}$ & $\phi_{2}$ & $\begin{array}{c}\psi \\
\text { (degree) }\end{array}$ \\
\hline (a) Individual observing seasons & & & & \\
2009 & 54941.7 & 13 & $-0.359(8)$ & 17 & $0.187(6)$ & $30.9 \pm 1.7$ \\
$2009 / 10$ & 55200.4 & 16 & $-0.357(5)$ & 14 & $0.191(3)$ & $30.0 \pm 1.1$ \\
$2010 / 11$ & 55624.7 & 13 & $-0.356(9)$ & 12 & $0.189(4)$ & $29.9 \pm 1.7$ \\
$2011 / 12$ & 55932.6 & 14 & $-0.368(8)$ & 11 & $0.188(6)$ & $32.3 \pm 1.7$ \\
2013 & 56333.6 & 8 & $-0.367(12)$ & 3 & $0.189(12)$ & $32.1 \pm 3.0$ \\
(b) All data combined & & & & & \\
$2009-2013$ & 55478.2 & 64 & $-0.361(9)$ & 57 & $0.189(5)$ & $31.0 \pm 1.9$ \\
\hline
\end{tabular}

Notes. Phase errors quoted in parentheses refer to the last digits.

trinsic scatter, possibly caused by emission from the second pole. In the statistical analysis, the isolated value $\phi_{1}=-0.414$ in Fig. 2 (left panel) was excluded. It was observed on 12 February 2013, close in time to the abnormal event of Fig. 1 (bottom panel).

\subsection{Temporal variation of the longitude of the prime accretion spot}

The mean values of $\phi_{1}$ and $\phi_{2}$ for the five individual observing seasons and for all seasons combined are listed in Table 1 along with the corresponding longitudes of the prime-pole accretion spot, $\psi=\left(\phi_{2}-\phi_{1}\right) \times 180^{\circ}$. This longitude is essentially constant over the 2009-2013 period, despite variations in the accretion rate $\dot{M}$. This result indicates that wandering of the accretion spot across the surface of the white dwarf as a response to $\dot{M}$ variations are unimportant in the present context. That $\dot{M}$ does not significantly affect the spot longitude was previously noted for instance by Schwope et al. (2002). For completeness, we summarize the previously published spot longitudes in Table 2. Figure 3 shows the time variation of the spot longitude. The published values are displayed as green circles, the new ones as cyan-blue filled circles. Our measurements deviate from the extrapolation of the pre-2003 linear increase (dashed line) by highly significant $\sim 11^{\circ}$ in 2009 and $\sim 18^{\circ}$ in 2013 . We conclude that the secular progression of the spot longitude has halted, and that the combined data are inconsistent with the notion mv of an asynchronously rotating white dwarf (dashed line).

We have tested the alternative model of an oscillation of the magnetic axis of the white dwarf about an equilibrium position by fitting a sinusoid to the data (solid curve). The fit has a $\chi^{2}=$ 6.8 for 12 degrees of freedom and yields a period of $60 \pm 9 \mathrm{yr}$, an amplitude of $25^{\circ} \pm 3^{\circ}$, and an equilibrium orientation of $7^{\circ} \pm$ $3^{\circ}$. The fit plausibly explains all available data on the temporal variation of the spot longitude.

\section{Discussion and conclusion}

We have, for the first time, presented evidence for the oscillation or libration of the magnetic axis of the white dwarf in a polar that results from the combined action of the accretion and magnetic torques. The theory of the magnetostatic interaction of the magnetic moments of white dwarf and the secondary star predicts an oscillation period on the order of a few decades and an amplitude
Table 2. Previously published prime-pole spot longitudes $\psi$.

\begin{tabular}{lccrl}
\hline \hline Year & $\begin{array}{c}\text { Mean JD } \\
2450000+\end{array}$ & Band & $\begin{array}{c}\psi \\
\text { (degree) }\end{array}$ & Reference \\
\hline 1979 & 44214.62 & $\mathrm{X}$ & $-22.0 \pm 11.0$ & Biermann et al. (1985) \\
1982 & 44993.96 & Opt & $-14.0 \pm 7.0$ & \\
1984 & 45763.83 & Opt & $-14.0 \pm 4.0$ & \\
1984 & 45854.88 & $\mathrm{X}$ & $-12.0 \pm 7.0$ & Robinson \& Córdova (1984) \\
1985 & 46054.94 & $\mathrm{X}$ & $-11.0 \pm 7.0$ & \\
1991 & 48560.56 & FUV & $3.0 \pm 4.0$ & Stockman et al. (1994) \\
1992 & 48686.50 & Opt & $4.0 \pm 3.0$ & Bailey et al. (1993) \\
1992 & 48773.22 & $\mathrm{X}$ & $2.2 \pm 2.2$ & Schwope et al. (2002) \\
1993 & 49144.09 & $\mathrm{X}$ & $4.7 \pm 2.2$ & \\
2000 & 51870.84 & $\mathrm{X}$ & $24.1 \pm 2.2$ & \\
2000 & 51870.84 & $\mathrm{X}$ & $25.0 \pm 1.0$ & Pandel et al. (2002) \\
2002 & 52284.70 & Opt & $26.6 \pm 3.0$ & Schwope et al. (2002) \\
& & & & and this work \\
\hline
\end{tabular}

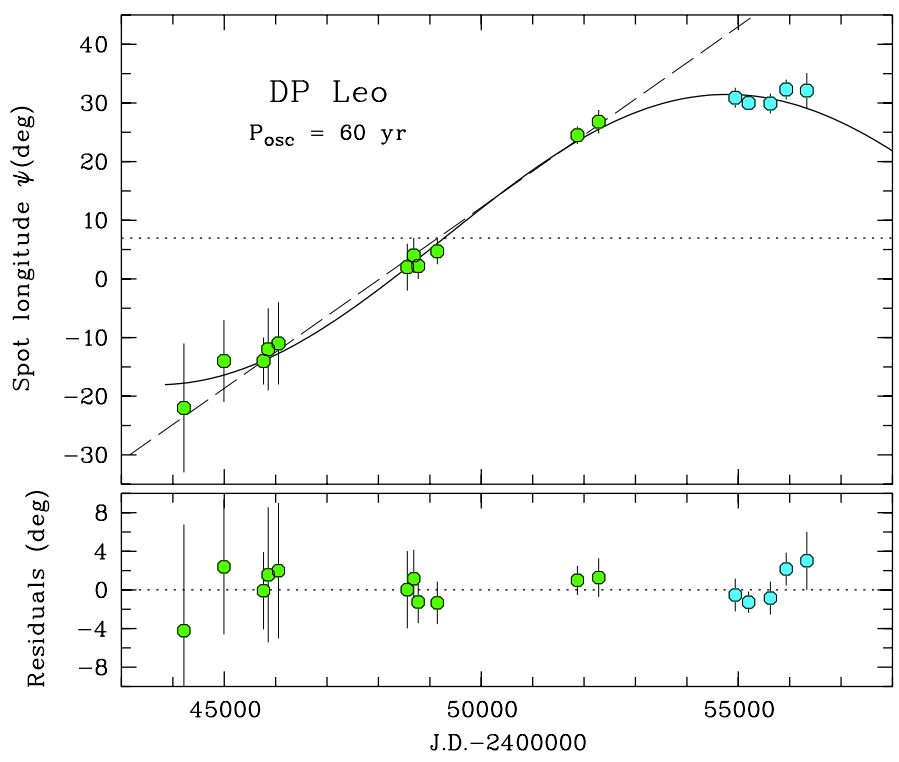

Fig. 3. Top: time dependence of the longitude of the prime-pole accretion spot. Bottom: residuals from the sinusoidal fit.

of a few tens of degrees about an equilibrium longitude of the accreting pole that slightly leads the line connecting the stars (King et al. 1990; King \& Whitehurst 1991; Wickramasinghe \& Wu 1991; Wu \& Wickramasinghe 1993; Campbell \& Schwope 1999). A period of about $60 \mathrm{yr}$, an amplitude of $25^{\circ}$, and an equilibrium longitude of about $7^{\circ}$ are entirely consistent with theory. Schwope et al. (2002) had argued that with increasing $\psi$ the second pole becomes more easily accessible for the accretion stream. The enhanced cyclotron emission detected by us from the second pole is in line with this argument. If the magnetic axis of the white dwarf in DP Leo is, in fact, turning back, we predict that second-pole emission again becomes rare in the years to come. We caution, however, that the data cover only one half of the implied oscillation period, and we do not claim to have presented final proof of the proposed oscillation. Proving the periodicity and the existence of a secularly stable equilibrium position requires several more decades of monitoring of DP Leo.

Acknowledgements. Our data were obtained with the MONET/North telescope of the MOnitoring NEtwork of Telescopes, funded by the Alfried Krupp 
von Bohlen und Halbach Foundation, Essen, and operated by the Georg-AugustUniversität Göttingen, the McDonald Observatory of the University of Texas at Austin, and the South African Astronomical Observatory. The "Astronomie \& Internet" program of the Foundation and the MONET consortium provide observation time to astronomical projects in high-schools worldwide. Part of the early data were obtained in collaboration with Jens Diese and his high-school students at the Max-Planck-Gymnasium, Göttingen, Germany.

\section{References}

Bailey, J., Wickramasinghe, D. T., Ferrario, L., Hough, J. H., \& Cropper, M. 1993, MNRAS, 261, L31

Beuermann, K., Buhlmann, J., Diese, J., et al. 2011, A\&A, 526, A53

Biermann, P., Schmidt, G. D., Liebert, J., et al. 1985, ApJ, 293, 303

Campbell, C. G., \& Schwope, A. D. 1999, A\&A, 343, 132
Cropper, M., \& Wickramasinghe, D. T. 1993, MNRAS, 260, 696

Eastman, J., Siverd, R., \& Gaudi, B. S. 2010, PASP, 122, 935

King, A. R., \& Whitehurst, R. 1991, MNRAS, 250, 152

King, A. R., Whitehurst, R., \& Frank, J. 1990, MNRAS, 244, 731

Pandel, D., Cordova, F. A., Shirey, R. E., et al. 2002, MNRAS, 332, 116

Qian, S.-B., Liao, W.-P., Zhu, L.-Y., \& Dai, Z.-B. 2010, ApJ, 708, L66

Robinson, C. R., \& Cordova, F. A. 1994, ApJ, 437, 436

Schaaf, R., Pietsch, W., \& Biermann, P. 1987, A\&A, 174, 357

Schmidt, G. D., \& Stockman, H. S. 1991, ApJ, 371, 749

Schwope, A. D., Hambaryan, V., Schwarz, R., Kanbach, G., \& Gänsicke, B. T. 2002, A\&A, 392, 541

Stockman, H. S., Schmidt, G. D., Liebert, J., \& Holberg, J. B. 1994, ApJ, 430, 323

Wickramasinghe, D. T., \& Wu, K. 1991, MNRAS, 253, 11

Wu, K., \& Wickramasinghe, D. T. 1993, MNRAS, 260, 141 